\title{
Approaching the Ground State of the Kagomé Antiferromagnet
}

\author{
W. Schweika, ${ }^{1}$ M. Valldor, ${ }^{2, *}$ and P. Lemmens ${ }^{2}$ \\ ${ }^{1}$ Institut für Festkörperforschung, Forschungszentrum Jülich, D-52425 Jülich, Germany \\ ${ }^{2}$ Physik der Kondensierten Materie, TU-Braunschweig, D-38106 Braunschweig, Germany
}

(Received 10 July 2006; published 7 February 2007)

\begin{abstract}
$\mathrm{Y}_{0.5} \mathrm{Ca}_{0.5} \mathrm{BaCo}_{4} \mathrm{O}_{7}$ contains kagomé layers of $\mathrm{Co}$ ions, whose spins are strongly coupled, with a CurieWeiss temperature of $-2200 \mathrm{~K}$. At low temperature, $T=1.2 \mathrm{~K}$, our diffuse neutron scattering study with polarization analysis reveals characteristic spin correlations close to a predicted two-dimensional coplanar ground state with staggered chirality. The absence of three-dimensional long-range antiferromagnetic order indicates negligible coupling between the kagomé layers. The scattering intensities are consistent with high spin $S=3 / 2$ states of $\mathrm{Co}^{2+}$ in the kagomé layers and low spin $S=0$ states for $\mathrm{Co}^{3+}$ ions on interlayer sites. Our observations agree with previous Monte Carlo simulations indicating a ground state of effectively short range, staggered chiral spin order.
\end{abstract}

DOI: 10.1103/PhysRevLett.98.067201

The topology of many crystal structures has an important influence on the collective behavior of interacting magnetic moments. In lattices with triangular networks the antiferromagnetic (AF) coupling between all spins cannot be satisfied simultaneously owing to geometrical frustration that strongly reduces the ordering temperature and disturbs the settling of the system into a long-range ordered state. Exotic phenomena such as spin-ice and spinliquid phases can emerge from magnetic interactions that are incompatible with the underlying crystal geometry. A hallmark of frustration is the large degeneracy of complex, noncollinear ground states of finite entropy and the appearance of chiral correlations [1]. According to the MerminWagner theorem [2], in low dimensions, the AF order that would reveal the ground state properties is suppressed at finite temperatures. The famous case of spins on the twodimensional kagomé lattice that are simply AF coupled to only nearest neighbor comprises high geometrical frustration and low dimensionality and still challenges theoretical understanding as well as experimental observations and analysis.

Here, we present diffuse neutron scattering results with polarization analysis on a new compound $\mathrm{Y}_{0.5} \mathrm{Ca}_{0.5} \mathrm{BaCo}_{4} \mathrm{O}_{7}$, in which the Co spins $(S=3 / 2)$ in noninteracting kagomé layers appear to realize ideally the kagomé AF with only 2D nearest neighbor interactions, which allows an unprecedented approach to its unusual ground state properties.

Considering the classical Heisenberg model of $\mathrm{AF}$ coupled nearest neighbor spins on the kagomé lattice, the ground state is highly degenerate and characterized by competing chiral spin structures. The spins have a relative orientation of 120 degrees in each triangular unit, so that the local sum of spins is zero. However, there are two competing structures that show either uniform or staggered chirality, see Fig. 1. According to predictions for the classical Heisenberg AF the staggered chiral structure is favored $[3,4]$, because the existence of local zero energy modes, so-called weathervane defects, which are common
PACS numbers: 75.25.+z, 61.12.-q, 75.40.Cx, 75.50.Ee

spin rotations on hexagons, cause a larger degeneracy; such entropical selection follows the principle of order by disorder [5]. According to Monte Carlo (MC) simulations, domain walls in the staggered chiral structure are moved by weathervane defects, which apparently destroy the long-range chiral order [4] and may give rise to residual entropy in analogy to ice [6] and spin-ice systems [7]. Quantum fluctuations also favor the staggered chiral structure [8]. However, it is not clear whether the ground state is long-range ordered. The macroscopic classical ground state degeneracy could be removed by quantum fluctuations [9]; on the other hand, for $S=1 / 2$ spin dimers in the singlet state [10] a disordered quantum spin-liquid ground state is expected.

Experimentally, it is difficult to approach the ground state of the kagomé AF. At low but finite temperature, the specific disorder predicted for the classical AF causes relaxations that do not freeze-in. The spin correlations can
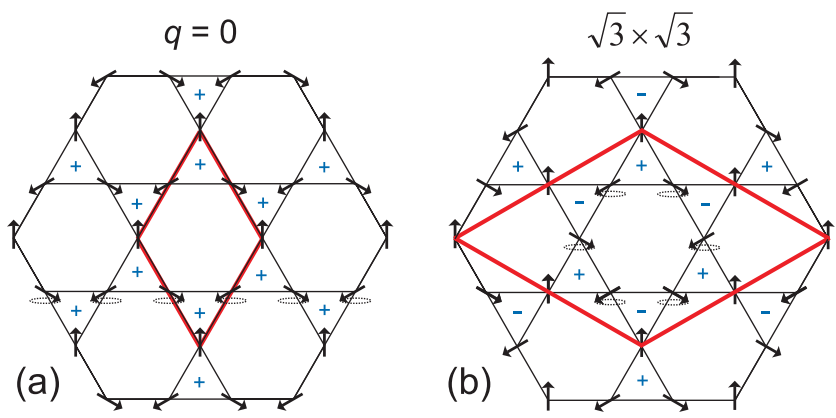

FIG. 1 (color). Infinitely degenerate AF ground states on the kagomé lattice. (a) The $q_{0}$ structure with uniform chirality; (b) the staggered chiral structure, which has a larger unit cell by $\sqrt{3} \times \sqrt{3}$. The chirality is denoted by the sign inside the triangles; chirality is positive when spins rotate by +120 degrees as one goes anticlockwise around a triangle. The dashed ellipses represent thermal fluctuations of zero energy: (a) common spin rotations in an infinite chain; (b) the weathervane defect, a common local spin rotation on a hexagon around the axis of the spins on the edges of the unit cell. 
be measured by quasielastic neutron scattering; however, this requires bulk materials that realize this twodimensional system. So far, such experimental evidence of absence of long-range order and spin-liquid behavior with spin correlations of two-dimensional character has been found [11,12], although results are not conclusive for the ground state properties of the kagomé AF. The material of Ref. [11], $\mathrm{SrCr}_{8-x} \mathrm{Ga}_{4+x} \mathrm{O}_{19}$ (SCGO), is in fact a bilayer system with inherent spin dilution. In a recent study of $\mathrm{KFe}_{3}\left(\mathrm{SO}_{4}\right)_{2}(\mathrm{OD})_{6}$ [13], significant interlayer coupling leads to $3 \mathrm{D}$ ordering below $65 \mathrm{~K}$, which prevents a close approach to the ground state. Furthermore, unexpected preference for uniform chirality is observed, which indicates the relevance of interactions to next-nearest neighbors, a fact supported by a recent measurement of the low energy spin wave excitations [14].

In the present study we investigate a new material, $\mathrm{Y}_{0.5} \mathrm{Ca}_{0.5} \mathrm{BaCo}_{4} \mathrm{O}_{7}$, see Fig. 2, with $\mathrm{Co}^{2+}$ and $\mathrm{Co}^{3+}$ mixed valence [15]. In this class of materials [16], with symmetry $\mathrm{P6}_{3} \mathrm{mc}$, Co occupies two different tetrahedral sites: $75 \%$ of all $\mathrm{Co}(\mathrm{Co} 2)$ constitute the kagomé layers and the remaining 25\% fill the Co1 sites, which are located on threefold axes between the kagomé layers. Shorter Co-O distances indicate a larger valence of $\mathrm{Co} 1$ than of $\mathrm{Co} 2$, and a preference for $\mathrm{Co}^{3+}$. A first indication for high spin frustration and possible 2D character of the spin correlations stems from susceptibility measurements that yield a Curie-Weiss temperature of $-2200 \mathrm{~K}$, indicating a huge AF exchange, without any evidence for a phase transition down to $4 \mathrm{~K}$.

Polycrystalline $\mathrm{Y}_{0.5} \mathrm{Ca}_{0.5} \mathrm{BaCo}_{4} \mathrm{O}_{7}$ samples for the diffuse neutron scattering experiments have been synthesized by a solid-state reaction in air [15]. The oxygen stoichiometry should be ideal as verified by iodometric titration on the parent compound $\mathrm{YBaCo}_{4} \mathrm{O}_{7}$ [17]. The scattering experiments were performed on the diffuse neutron scattering instrument DNS in Jülich. The magnetic scattering

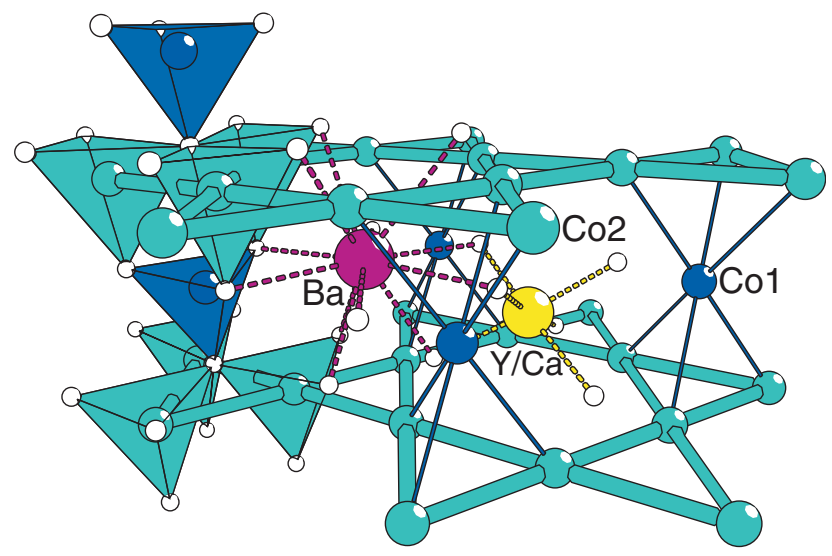

FIG. 2 (color). The $\mathrm{Y}_{0.5} \mathrm{Ca}_{0.5} \mathrm{BaCo}_{4} \mathrm{O}_{7}$ crystal structure. Thicker bonds between the $\mathrm{Co} 2$ atoms highlight the kagomé layers. All Co atoms are tetrahedrally coordinated by oxygen, as shown in the polyhedral representation (left), emphasizing the threefold symmetry axis, going through Co1, which allow trimerization of $\mathrm{Co} 2$ in the kagomé substructure. was separated from nuclear background scattering by polarization analysis. The separated high background due to nuclear spin-incoherent scattering from Co serves as an intrinsic calibration of the paramagnetic cross section, a calibration that avoids systematic errors and corrections. A strong diffuse peak gradually emerges and sharpens upon cooling and its asymmetric shape indicates low dimensional spin correlations, see Fig. 3. Remarkably, no longrange magnetic order appears even at $1.2 \mathrm{~K}$. The magnetic scattering can be described in terms of the Fourier transform of the spin pair-correlations [18], where we neglect a possible relationship between spin correlation and spin direction:

$$
\frac{d \sigma}{d \Omega_{\mathrm{mag}}}(Q)=\frac{2}{3} r_{0}^{2} F_{Q}^{2} \sum_{r}\left\langle\vec{S}_{0} \cdot \vec{S}_{r}\right\rangle \frac{\sin Q r}{Q r},
$$

where $Q$ is the modulus of the scattering vector, $r_{0}=$ $-0.54 \times 10^{-12} \mathrm{~cm}$ the magnetic scattering length, $S$ the spin quantum number of the scattering ion, and $F_{Q}$ the magnetic scattering form factor of a single $\mathrm{Co}^{2+}$ ion. For an ideal paramagnet, the scattering is purely elastic and proportional to the self-correlation $S(S+1)$. In general, one has to consider the expectation value of the timedependent self-correlation function of a spin precessing in the strong local field of its neighbors. Here, the expectation value is reduced to the scalar product of the ordered moments $\left\langle S_{z}^{2}\right\rangle$ because thermal energies are small compared to the exchange energy and the signal is integrated only over small energy transfers.

Additional time-of-flight experiments without neutron polarization analysis show that the scattering at $1.2 \mathrm{~K}$ is essentially elastic; i.e., spin correlations appear to be frozen within the energy resolution of $0.5 \mathrm{meV}$. Remarkably, with increasing temperature the dynamic response is gapless and the change in scattering is essentially due to

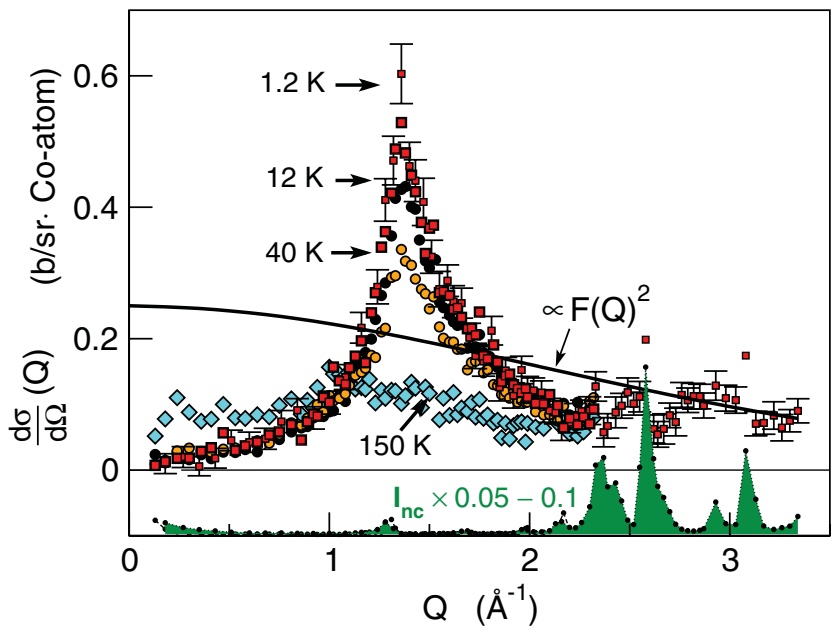

FIG. 3 (color). Temperature dependence of the diffuse magnetic scattering; data are calibrated by the spin-incoherent scattering of $\mathrm{Co}(0.382 \mathrm{~b} / \mathrm{sr})$; the separated nuclear coherent scattering, $\mathbf{I}_{n c}$, has been rescaled and shifted (bottom part). 


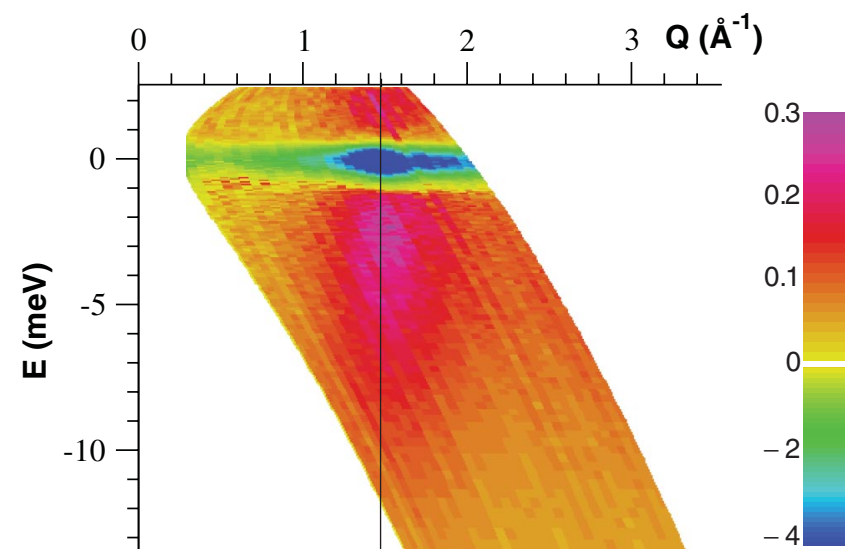

FIG. 4 (color). Quasielastic broadening due to thermal spin fluctuations. The difference of $S(Q, \omega)$ measured at $150 \mathrm{~K}$ and $40 \mathrm{~K}$ in time-of-flight mode reveals a loss of elastic intensity (blue) and a relaxation response (red) with a $Q$ dependence consistent with weathervane modes. The vertical line corresponds to the peak of model (iv) in Fig. 5.

quasielastic broadening, as seen in the intensity difference of Fig. 4, which essentially eliminates the structural scattering. Because both chiral structures (Fig. 1) are coplanar with strong in-plane AF exchange, the lowest dynamic response are relaxations out of the kagomé plane including possible zero energy modes. Note that interactions to further than nearest neighbors would raise these modes to a finite energy [14].

The magnetic scattering intensity allows us to estimate the average Co spin moment. A fit to the $1.2 \mathrm{~K}$ data yields a forward scattering $F S=0.25(2) \mathrm{b} /(\mathrm{srCo})$ for the selfcorrelation term. This intensity, which describes a random spin configuration, is depicted by a black line $\left[\propto F(Q)^{2}\right]$ in Fig. 3. The best quantitative agreement is found if we assume that $\mathrm{Co}^{2+}$ ions $(5 / 8$ of all $\mathrm{Co})$ are in high spin state $S=3 / 2$ and that $\mathrm{Co}^{3+}$ ions (3/8 of all Co) are in low spin state $S=0$ yielding FS $=0.273 \mathrm{~b} /(\mathrm{srCo})$. Clearly, $S=0$ at the Co1 sites rationalizes the absence of any significant interlayer coupling and, hence, the ultimate suppression of 3D long-range order. However, this configuration requires a fraction of $1 / 6$ of $\mathrm{Co}^{3+}$ ions on the kagomé sites. According to formal valencies an ideal site separation should be realized in the parent compound $\mathrm{YBaCo}_{4} \mathrm{O}_{7}$, which would lead to a closer realization of 2D magnetic behavior. However, $\mathrm{YBaCo}_{4} \mathrm{O}_{7}$ exhibits 3D long-range order [19] indicating possible covalence effects. In $\mathrm{Y}_{0.5} \mathrm{Ca}_{0.5} \mathrm{BaCo}_{4} \mathrm{O}_{7}$, an effective oxygen valence of 1.93 may restore the ideal $\mathrm{Co}^{2+}$ to $\mathrm{Co}^{3+}$ ratio of three. This would lead to slightly higher than observed scattering intensity, FS $=0.328 \mathrm{~b} /(\mathrm{srCo})$, but we do not rule out this possibility, because the scattering might not reveal the full ordered moment. $\mathrm{Co}^{3+}$ is situated closer to a trigonal plane changing the point-group symmetry from $T_{d}$ to $C_{3 v}$. According to Hund's rules, an ideal tetrahedral coordination with weak crystal field splitting, typical for oxygen ligands, results in high spin states, but low spin states, here $S=0$ at Co1 sites, are possible for $C_{3 v}$ symmetry [20].

The wave-vector dependence of the diffuse scattering provides direct information about the spin correlations, which impose constraints on possible chiral spin correlations, correlations that cannot be directly observed. The $1.2 \mathrm{~K}$ normalized diffuse magnetic scattering $S(Q)=\sum_{r}\left\langle\hat{S}_{0} \cdot \hat{S}_{r}\right\rangle \frac{\sin Q r}{Q r}$, where $\hat{S}$ are unit length spins, is shown in Fig. 5. We can immediately rule out spin correlations of the $q_{0}$ structure. The strongest peak would be at $Q=\left|\mathbf{q}_{0}\right|=\left|a^{*}(1,0)\right|=1.152 \AA^{-1}$, where $a^{*}=(2 \pi / a)(2 / \sqrt{3})$. This means that there is neither AF next-nearest neighbor coupling nor ferromagnetic (FM) coupling to third nearest neighbors that would stabilize the $q_{0}$ structure. Instead, the observed peak coincides with $Q=\left|2 \mathbf{q}_{\sqrt{3}}\right|=\left|2 a^{*}(1 / 3,1 / 3)\right|=1.330 \AA^{-1}$, a reciprocal lattice vector of the $\sqrt{3} \times \sqrt{3}$ structure. The data were also analyzed in terms of spin correlations by a Fourier analysis for distances on the kagomé lattice using a linear least squares refinement. The results are shown in the inset of Fig. 5, where the spin correlations $\left\langle\hat{S}_{0} \cdot \hat{S}_{r}\right\rangle$ have been normalized to the correlation function $\cos \mathbf{q}_{\sqrt{3}} \cdot \mathbf{r}$, of the ideal $\sqrt{3} \times \sqrt{3}$ structure, and $G_{\sqrt{3}}(r)=$ $\left\langle\hat{S}_{0} \cdot \hat{S}_{r}\right\rangle / \cos \mathbf{q}_{\sqrt{3}} \cdot \mathbf{r}$. All parameters are positive, showing the preference for the $\sqrt{3} \times \sqrt{3}$ structure, and decay to zero, as expected for the absence of long-range order.

The scattering of both structures, $q_{0}$ and $\sqrt{3} \times \sqrt{3}$, have been modeled with a suitable asymptotic decay function to the spin correlations, see Fig. 5. A power law decay of the

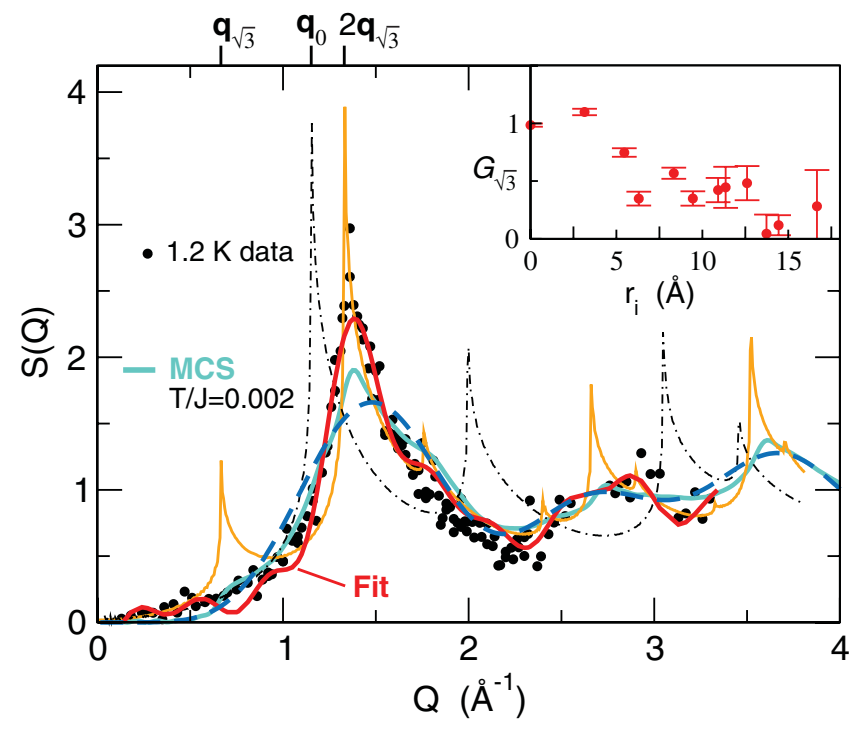

FIG. 5 (color). Modeling of $1.2 \mathrm{~K}$ scattering data. (i) Power law decay of spin correlations in the $q_{0}$ and $\sqrt{3} \times \sqrt{3}$ structures, respectively (dashed dotted black and solid orange lines); (ii) Fourier analysis (fit, red line), yielding $G_{\sqrt{3}}$ (inset) spin correlations normalized to those of the $\sqrt{3} \times \sqrt{3}$ structure with ideal staggered chirality; (iii) $\mathrm{MC}$ data (thick cyan line) for a classical Heisenberg $\mathrm{AF}$ at $T / J=0.002$ [4]; (iv) weathervane model (dashed blue line). 
2D spin correlations is expected close to the ground state $[3,4,21]$. A reasonable description of $S(Q)$ is found with the $\sqrt{3} \times \sqrt{3}$ model for a power law decay $\left\langle\hat{S}_{0} \cdot \hat{S}_{r}\right\rangle \propto r^{-\eta}$, with $\eta \approx 0.6$, which resembles the sharp cusp at top of this broad diffuse peak. However, the data are probably broadened by the experimental resolution, $\Delta Q / Q \approx 0.03$, a resolution that is not sufficient to clearly distinguish between power law or exponential decay of the spin correlations. The data are in good qualitative agreement with the classical 2D MC simulations. Note that the observed peak at $\left|2 q_{\sqrt{3}}\right|$, is significantly more pronounced than in the MC results, and indicates, in agreement with Ref. [4], a critical point at $T=0 \mathrm{~K}$ with true long-range order. The ground state is approached much closer in our experiment than in the MC simulations although temperature and Curie-Weiss constant of approximately $-2200 \mathrm{~K} \approx 4 J$ [15], where $J$ is the exchange energy, match closely the conditions of the simulation, $T / J=0.002$, which can be understood by the lack of ergodicity in the simulations at low temperature.

Surprisingly, the data do not show any indication of a peak at $Q=q_{\sqrt{3}}$ that should follow from the larger unit cell of the $\sqrt{3} \times \sqrt{3}$ structure, which reveals that the longrange ordered ground state must exhibit a specific type of disorder. A possible insight is given by the MC simulations of [4], where the $q_{\sqrt{3}}$ peak is also absent. In the simulations, the chiral spin correlations $\left\langle\hat{S}_{0} \times \hat{S}_{r}\right\rangle$ decay rapidly, which has been attributed to the weathervane defects allowing domain wall motion. An interesting question is whether our data can provide any further indication for the existence of the weathervane defects that seem to disturb the chiral long-range order of the ground state in the previous MC simulations. One simple defect model is to place the weathervane defects on two of the three magnetic sublattices. The configurational average of this model is equivalent to a collinear structure model and the correlations are reduced by a factor 2 with respect to the ideal structure for distances exceeding the spacings of the unit cell. The preserved short-range correlations combined with reduced long-range correlations lower the $q_{\sqrt{3}}$ peak relative to the $2 q_{\sqrt{3}}$ peak. Further disorder arises from flipping larger loops connecting two types of spins, a flipping that introduces domain walls and may rationalize the absence of the $q_{\sqrt{3}}$ peak as seen in the MC simulations. Any FM coupling between next-nearest spins would lift the weathervane degeneracy and induce correlations that would produce the ideally ordered structure with a peak at $q_{\sqrt{3}}$. Therefore, interactions to farther than nearest neighbors must be small compared to $k_{B} T$. The spin correlation for a weathervane hexagon and the related $S(Q)$ can easily be modeled; here, we add the AF coupling toward surrounding spins such that the sum of spins equals to zero. As shown in Fig. 5, model (iv), this yields a qualitatively good description of the measured $S(Q)$ confirming that the specific local chiral correlation of a spin hexagon is the relevant local feature of the structure. The quasielastic broadening at $150 \mathrm{~K}$ shown in Fig. 4 indicates relaxation rather than tunneling dynamics for the weathervane modes, and, even at low $T$, relaxation is expected due to destructive quantum interference and vanishing tunneling amplitudes for weathervane modes in half integer spin systems [22]. The emergence of hexagon spin loops has been observed previously in another highly frustrated, however 3D spin system, of $\mathrm{Cr} S=3 / 2$ spins in $\mathrm{ZnCr}_{2} \mathrm{O}_{4}$ [23].

In conclusion, the present neutron scattering study with polarization analysis on a new compound, realizing the $S=3 / 2$ kagome $\mathrm{AF}$, agrees in surprising detail with the complexity of the ground state of the classical Heisenberg AF for only nearest neighbor interactions. The remarkable survival of only short-range $2 \mathrm{D}$ spin correlations at low temperature results from low spin $S=0$ states of $\mathrm{Co}^{3+}$ ions at the interlayer sites, which decouple the kagomé layers. Despite the diverging range of spin correlations when approaching the ground state, the data indicate a short-range nature of chiral spin correlations, where the relevant feature is the weathervane mode of spin hexagons.

We thank C. L. Henley for useful comments and discussions, R. P. Hermann for a critical reading of the manuscript, and J. N. Reimers and A. J. Berlinsky for providing us with their original MC data.

*Present address: Institute of Physics 2, Physics Department, D-50937 Cologne, Germany.

[1] R. Moessner and A. T. Ramirez, Phys. Today 59, No. 2, 24 (2006).

[2] N. D. Mermin and H. Wagner, Phys. Rev. Lett. 17, 1133 (1966).

[3] D. A. Huse and A. D. Rutenberg, Phys. Rev. B 45, 7536 (1992).

[4] J. N. Reimers and A. J. Berlinsky, Phys. Rev. B 48, 9539 (1993).

[5] J. Villain et al., J. Phys. (Paris) 41, 1263 (1980).

[6] L. Pauling, The Nature of the Chemical Bond (Cornell University Press, Ithaca, NY, 1945).

[7] M. J. Harris et al., Phys. Rev. Lett. 79, 2554 (1997).

[8] S. Sachdev, Phys. Rev. B 45, 12377 (1992).

[9] T. Yildirim, Tr. J. of Phys. 23, 47 (1999).

[10] C. Zeng and V. Elser, Phys. Rev. B 51, 8318 (1995).

[11] C. Broholm, G. Aeppli, G. P. Espinosa, and A. S. Cooper, Phys. Rev. Lett. 65, 3173 (1990).

[12] A. S. Wills et al., Phys. Rev. B 64, 094436 (2001).

[13] D. Grohol et al., Nat. Mater. 4, 323 (2005).

[14] K. Matan et al., Phys. Rev. Lett. 96, 247201 (2006).

[15] M. Valldor, Solid State Sci. 8, 1272 (2006).

[16] M. Valldor and M. Andersson, Solid State Sci. 4, 923 (2002).

[17] M. Karppinen et al., Chem. Mater. 18, 490 (2006).

[18] I. A. Blech and B. L. Averbach, Physics (N.Y.) 1, 31 (1964).

[19] L. C. Chapon et al., Phys. Rev. B 74, 172401 (2006).

[20] E. K. Byrne et al., J. Chem. Soc. D 19, 1491 (1986); D. M. Jenkins et al., J. Am. Chem. Soc. 124, 15336 (2002).

[21] D. A. Garanin and B. Canals, Phys. Rev. B 59, 443 (1999).

[22] J. von Delft and C. L. Henley, Phys. Rev. Lett. 69, 3236 (1992).

[23] S.-H. Lee et al., Nature (London) 418, 856 (2002). 